\title{
HYPERLOGARITHMIC EXPANSION AND THE VOLUME OF A HYPERBOLIC SIMPLEX
}

\author{
K. AOMOTO \\ Department of Mathematics, Nagoya University \\ Nagoya, Japan
}

0. Introduction. Hyperlogarithmic functions (or higher logarithmic functions) are multivalued analytic functions defined on complex projective varieties, with unipotent monodromy and with regular singularity. It is known that they can be expressed by the use of iterated integrals of suitable logarithmic 1-forms in the sense of K. T. Chen (see [A1], [H1]). Recently these functions have played a considerable role in various problems of geometry and arithmetic (for example, see [H2], [B1], [G2], [V], etc.). These are a special case of hypergeometric functions on a Grassmannian manifold (see [A2], [G1], [V]).

However, there are other kinds of hyperlogarithmic functions which are related to the configuration of hyperplanes and a hyperquadric (see [A3]). The volume of a simplex in a hyperbolic space is a hyperlogarithmic function of basic algebraic invariants, as a simple consequence of the Schläfli formula. However, there remains the problem of divergence in the case where the vertices lie on the boundary.

In this note we want to derive a modified Schläfli formula in such a degenerate case and give a hyperlogarithmic expansion for the volume, by using a technique developed in [A3]. A similar result has been obtained by Kellerhals [K4]. Her method is to decompose a simplex into several orthoschemes and to obtain an explicit formula for each orthoscheme by using the Lobachevskii function $\pi(x)$.

In the appendix we discuss a relation between the volume and Appell's hypergeometric functions of type $F_{4}$.

1. The Schläfli formula. A geodesic simplex $\Delta$ in the $n$-dimensional hyperbolic space $H=\left\{t_{0}^{2}-t_{1}^{2}-\ldots-t_{n}^{2}=1, t_{0}>0\right\}$ is defined by the inequalities $f_{j}(t) \geq 0$ for $n+1$ linear functions $f_{j}(t)=u_{j, 0}+\sum_{\nu=1}^{n} u_{j, \nu} t_{\nu}, 1 \leq j \leq n+1$. Its 
volume $V_{n}(\Delta)$ is given by the integral

$$
V_{n}(\Delta)=\int_{f_{1} \geq 0, \ldots, f_{n+1} \geq 0} \Phi d t_{0} \wedge \ldots \wedge d t_{n+1}
$$

for $\Phi=\exp \left[-\frac{1}{2}\left(t_{0}^{2}-t_{1}^{2}-\ldots-t_{n}^{2}\right)\right]$. This is also equal to $2^{(n-1) / 2} \Gamma((n+1) / 2) V_{n}(\widehat{\Delta})$ for a geodesic simplex $\widehat{\Delta}$ in the disc $D=\left\{x_{1}^{2}+\ldots+x_{n}^{2}<1\right\}$ defined by the inequalities $\widehat{f}_{j}(x) \geq 0$ for the inhomogeneous linear functions $\widehat{f}_{j}(x)=u_{j, 0}+$ $\sum_{\nu=1}^{n} u_{j, \nu} x_{\nu}$. The volume $V_{n}(\widehat{\Delta})$ is defined by the integral

$$
V_{n}(\widehat{\Delta})=\int_{\widehat{\Delta}}\left(1-x_{1}^{2}-\ldots-x_{n}^{2}\right)^{-(n+1) / 2} d x_{1} \wedge \ldots \wedge d x_{n} .
$$

First we assume that $\widehat{\Delta}$ lies in $D$. Then $u_{j, 0}^{2}-\sum_{\nu=1}^{n} u_{j, \nu}^{2}<0$. We may normalize it so that $u_{j, 0}^{2}-\sum_{\nu=1}^{n} u_{j, \nu}^{2}=-1$. Because of conformal invariance, $V_{n}(\Delta)$ or equivalently $V_{n}(\widehat{\Delta})$ depends only on the inner products $a_{j, k}=u_{j, 0} u_{k, 0}-\sum_{\nu=1}^{n} u_{j, \nu} u_{k, \nu}$ for $1 \leq j, k \leq n+1$. $a_{j, k}, j \neq k$, can also be expressed as $\cos \langle j, k\rangle$, where $\langle j, k\rangle$ denotes the dihedral angle subtended by $\widehat{\Delta}$ between the hyperplanes $F_{j}=\left\{\widehat{f}_{j}(x)=\right.$ $0\}$ and $F_{k}=\left\{\widehat{f}_{k}(x)=0\right\}$. We denote by $A$ the symmetric $(n+1) \times(n+1)$ matrix $\left(\left(a_{j, k}\right)\right)_{1 \leq j, k \leq n+1}$. Note that $a_{j, j}=-1$. We denote by $A\left(\begin{array}{c}i_{1} \ldots, i_{p} \\ j_{1}, \ldots, j_{p}\end{array}\right)$ the subdeterminant of $A$ with lines $i_{1}, \ldots, i_{p}$ and columns $j_{1}, \ldots, j_{p}$ for $\left\{i_{1}, \ldots, i_{p}\right\},\left\{j_{1}, \ldots, j_{p}\right\} \subset$ $\{1,2, \ldots, n+1\}$. We abbreviate $A\left(\begin{array}{c}i_{1} \ldots, i_{p} \\ j_{1}, \ldots, j_{p}\end{array}\right)$ to $A\left(i_{1}, \ldots, i_{p}\right)$.

One can show that $\widehat{\Delta}$ defines a simplex lying in $D$ if and only if

$$
\begin{aligned}
(-1)^{p} A\left(i_{1}, \ldots, i_{p}\right) & >0 \quad \text { for } 1 \leq p \leq n, \text { and } \\
(-1)^{n+1} A(1,2, \ldots, n+1) & <0 .
\end{aligned}
$$

We denote by $v_{1}, \ldots, v_{n+1}$ the vertices of $\widehat{\Delta}$ such that $v_{j} \in \widehat{\Delta} \cap F_{1} \cap \ldots \cap$ $F_{j-1} \cap F_{j+1} \cap \ldots \cap F_{n+1}$. Then $v_{j}$ is on the boundary $\partial D$ of $D$ if and only if $A(1,2, \ldots, j-1, j+1, \ldots, n+1)=0$. The Schläfli formula says that, as a function of the variables $a_{j, k}, V_{n}(\Delta)$ satisfies the variational formula

$$
d V_{n}(\widehat{\Delta})=-\frac{1}{2} \sum_{\substack{1 \leq j, k \leq n+1 \\ j \neq k}} V_{n-2}\left(\widehat{\Delta}_{j, k}\right) d\langle j, k\rangle,
$$

where $\widehat{\Delta}_{j, k}$ denotes the $(n-2)$-dimensional subsimplex $\widehat{\Delta}_{j, k}=\widehat{\Delta} \cap F_{j} \cap F_{k}$. $d\langle j, k\rangle$ is equal to the logarithmic 1 -form

$$
\theta\left(\begin{array}{c}
\emptyset \\
j, k
\end{array}\right)=\frac{1}{2 i} d \log \left(\frac{a_{j, k}+i \sqrt{A(j, k)}}{a_{j, k}-i \sqrt{A(j, k)}}\right) .
$$

Further, for $I=\left\{i_{1}, \ldots, i_{p}\right\}$ and $J=\left\{i_{1}, \ldots, i_{p}, i_{p+1}, i_{p+2}\right\}$ we define the loga- 
rithmic 1-form

$$
\theta\left(\begin{array}{l}
I \\
J
\end{array}\right)=\frac{1}{2 i} d \log \left(\frac{A\left(\begin{array}{l}
I, i_{p+1} \\
I, i_{p+2}
\end{array}\right)+i \sqrt{A(I) A(J)}}{A\left(\begin{array}{l}
I, i_{p+1} \\
I, i_{p+2}
\end{array}\right)-i \sqrt{A(I) A(J)}}\right)
$$

for $p \leq n-2$, and

$$
\theta\left(\begin{array}{l}
I \\
J
\end{array}\right)=\frac{1}{2} d \log \left(\frac{A\left(\begin{array}{c}
I, i_{n} \\
I, i_{n+1}
\end{array}\right)+\sqrt{-A(I) A(J)}}{A\left(\begin{array}{c}
I, i_{n} \\
I, i_{n+1}
\end{array}\right)-\sqrt{-A(I) A(J)}}\right)
$$

for $p=n-1, n$ odd.

As a simple consequence of $(1.5), V_{n}(\widehat{\Delta})$ can be expressed as a hyperlogarithm (sometimes called higher logarithm) (see $[\mathrm{A} 5])$ :

$$
V_{n}(\widehat{\Delta})=\sum_{\emptyset \subset I_{1} \subset \ldots \subset I_{\nu}} \int_{*}^{A} \theta\left(\begin{array}{c}
\emptyset \\
I_{1}
\end{array}\right) \ldots \theta\left(\begin{array}{c}
I_{\nu-1} \\
I_{\nu}
\end{array}\right),
$$

for a sequence of increasing subsets $I_{1}, \ldots, I_{\nu}$ of $\{1,2, \ldots, n+1\}, I_{r}=\left\{i_{1}, \ldots\right.$ $\left.\ldots, i_{2 r}\right\} . \quad \nu$ is equal to $(n+1) / 2$ or $n / 2$ according as $n$ is odd or even. The integration on the right hand side means K. T. Chen's iterated integrals along a path from the base point $*$ to $A$. As special cases we have

$$
V_{1}(\widehat{\Delta})=\int_{\alpha}^{\beta}\left(1-x^{2}\right)^{-1} d x=\frac{1}{2} \log \frac{a_{1,2}+\sqrt{a_{1,2}^{2}-1}}{a_{1,2}-\sqrt{a_{1,2}^{2}-1}}
$$

for $\alpha=-u_{1,0} / u_{1,1}, \beta=-u_{2,0} / u_{2,1}$ and $a_{1,2}=u_{1,0} u_{2,0}-u_{1,1} u_{2,1}$, while

$$
V_{2}(\widehat{\Delta})=\pi-\langle 1,2\rangle-\langle 2,3\rangle-\langle 3,1\rangle \text {. }
$$

The following is an immediate consequence of (1.7).

LEMMA 1. The hyperbolic distance between $v_{n}$ and $v_{n+1}$ is given by

$$
\frac{1}{2} \log \frac{A\left(\begin{array}{c}
1, \ldots, n-1, n \\
1, \ldots, n-1, n+1
\end{array}\right)+\sqrt{A(1, \ldots, n-1) A(1, \ldots, n+1)}}{A\left(\begin{array}{c}
1, \ldots, n-1, n \\
1, \ldots, n-1, n+1
\end{array}\right)-\sqrt{A(1, \ldots, n-1) A(1, \ldots, n+1)}} .
$$

We see at the same time that

$$
A\left(\begin{array}{c}
i_{1}, \ldots, i_{n-1}, i_{n} \\
i_{1}, \ldots, i_{n-1}, i_{n+1}
\end{array}\right)>0
$$

This inequality will be used later for $n=3$.

2. Regularization of divergent integrals. When one of the vertices lies on $\partial D, V_{n}(\Delta)$ is well defined and continuous in $a_{j, k}$, while $V_{1}(\Delta)$ diverges. The formula (1.5) holds for $n \geq 4$ but not for $n=3$. We want to derive a modified version of the Schläfli formula for $V_{3}(\Delta)$. To do this, we use the technique of regularization of divergent integrals which has been frequently used since the 
times of J. Hadamard. We consider the integral

$$
V_{n}(\widehat{\Delta} \mid \mu)=\int_{\widehat{\Delta}}\left(1-|x|^{2}\right)^{-(n+1+\mu) / 2} d x_{1} \wedge \ldots \wedge d x_{n},
$$

for $\mu>0$. When $\mu=0$, it reduces to $V_{n}(\widehat{\Delta})$. (2.1) is no more conformally invariant. It cannot be expressed as a function of the variables $a_{j, k}$ for $1 \leq j, k \leq n+1$. We denote by $\widetilde{A}$ the enlarged symmetric $(n+2) \times(n+2)$ matrix $\left(\left(a_{j, k}\right)\right)_{0 \leq j, k \leq n+1}$ with $a_{0,0}=1$, and $a_{0, j}=a_{j, 0}=u_{j, 0}$. Obviously $a_{j, 0}$ is not conformally invariant.

The following variational formula has been proved in [A3] (see the formula (3.7) loc. cit. for $\left.\lambda_{1}, \ldots, \lambda_{n+1} \rightarrow 0\right)$ :

LEMma 2.1. For an arbitrary $n \geq 1$,

$$
\begin{aligned}
(n-1+\mu) d & V_{n}(\widehat{\Delta} \mid \mu) \\
= & -\frac{1}{2} \sum_{\substack{1 \leq j, k \leq n+1 \\
j \neq k}} d\langle j, k\rangle\left\{\frac{A(j, k)}{A(0, j, k)}\right\}^{-\mu / 2} V_{n-2}\left(\widehat{\Delta}_{j, k} \mid \mu\right) \\
& +\mu \sum_{k=1}^{n+1} d a_{0, k}\left\{\frac{-1}{A(0, k)}\right\}^{-\mu / 2} \frac{1}{\sqrt{A(0, k)}} V_{n-1}\left(\widehat{\Delta}_{k} \mid \mu-1\right),
\end{aligned}
$$

where $\widehat{\Delta}_{j, k}=\widehat{\Delta} \cap F_{j} \cap F_{k}$ and $\widehat{\Delta}_{k}=\widehat{\Delta} \cap F_{k} . V_{1}\left(\widehat{\Delta}_{j, k} \mid \mu\right)$ has a definite meaning and gives a function meromorphic in $\mu$ at least with a pole at $\mu=0$.

The following lemma can be seen by a computation.

Lemma 2.2. $A(0, i)<0, A(0, i, j)>0, A(0, i, j, k)<0$ for any $i, j, k \in$ $\{1,2,3,4\}$ and $A(0,1,2,3,4)=0$.

When $\beta=1$ in (1.9), $V_{1}(\widehat{\Delta} \mid \mu)$ has a Laurent expansion at $\mu=0$ :

$$
V_{1}(\widehat{\Delta} \mid \mu)=-\frac{1}{\mu}+\left\{\log 2-\frac{1}{2} \log \frac{1+\alpha}{1-\alpha}\right\}+O(\mu),
$$

with $\alpha=-a_{0,2} / \sqrt{-A(0,2)}$, where the constant term (denoted by C.T. $V_{1}(\widehat{\Delta} \mid \mu)$ ) represents the regular part of the divergent integral $V_{1}(\widehat{\Delta})$ :

$$
\operatorname{reg} V_{1}(\widehat{\Delta})=\text { C.T. } V_{1}(\widehat{\Delta} \mid \mu)=\log 2-\frac{1}{2} \log \frac{1+\alpha}{1-\alpha} .
$$

When $\alpha=\beta=-1$, we have

$$
V_{1}(\widehat{\Delta} \mid \mu)=-\frac{2}{\mu}+2 \log 2+O(\mu),
$$

whence $\operatorname{reg} V_{1}(\widehat{\Delta})=2 \log 2$.

3. Modified Schläfli formula for $n=3$. Because of symmetry, we only have to consider the following 4 cases: (i) $v_{4} \in \partial D$, (ii) $v_{3}, v_{4} \in \partial D$, (iii) $v_{2}, v_{3}, v_{4} \in \partial D$ and (iv) $v_{1}, v_{2}, v_{3}, v_{4} \in \partial D$. 
(1) Assume that $v_{4}$ lies on $\partial D$ and $v_{1}, v_{2}, v_{3} \in D$. This is equivalent to saying that $A(1,2,3)=0$, i.e. $\langle 1,2\rangle+\langle 2,3\rangle+\langle 3,1\rangle=\pi$. Then $V_{1}\left(\widehat{\Delta}_{1,2}\right), V_{1}\left(\widehat{\Delta}_{2,3}\right)$ and $V_{1}\left(\widehat{\Delta}_{3,1}\right)$ diverge, while $V_{1}\left(\widehat{\Delta}_{1,4}\right) V_{1}\left(\widehat{\Delta}_{2,4}\right), V_{1}\left(\widehat{\Delta}_{3,4}\right)$ are well defined. For $j, k=$ $1,2,3$, as $\mu$ tends to 0 , the coefficient of $\langle j, k\rangle$ on the right hand side of $(2.2)$ has a Laurent expansion

$$
\begin{aligned}
\left\{\frac{A(j, k)}{A(0, j, k)}\right\}^{-\mu / 2} V_{1}\left(\widehat{\Delta}_{j, k} \mid \mu\right) & \\
& =-\frac{1}{\mu}+\left\{\log 2-\frac{1}{2} \log \frac{1+\alpha}{1-\alpha}+\frac{1}{2} \log \frac{A(j, k)}{A(0, j, k)}\right\}+O(\mu),
\end{aligned}
$$

i.e.

$$
\begin{aligned}
\text { C.T. }\left[\left\{\frac{A(j, k)}{A(0, j, k)}\right\}^{-\mu / 2} V_{1}\left(\widehat{\Delta}_{j, k} \mid \mu\right)\right] \\
\quad=\log 2-\frac{1}{2} \log \frac{1+\alpha}{1-\alpha}+\frac{1}{2} \log \frac{A(j, k)}{A(0, j, k)} .
\end{aligned}
$$

Here $\alpha$ denotes $-A\left(\begin{array}{c}4, j, k \\ 0, j, k\end{array}\right) / \sqrt{-A(j, k) A(0, j, k, 4)}$. We set

$$
W_{j, k}=\frac{A(j, k)(1+\alpha)}{A(0, j, k)(1-\alpha)} .
$$

Then by taking the constant term of (2.2) in $\mu$, we have

$$
\begin{aligned}
2 d V_{3}(\widehat{\Delta})= & d\langle 1,2\rangle \log W_{1,2}+d\langle 2,3\rangle \log W_{2,3}+d\langle 3,1\rangle \log W_{3,1} \\
& +d\langle 1,4\rangle \log W_{1,4}+d\langle 2,4\rangle \log W_{2,4}+d\langle 3,4\rangle \log W_{3,4},
\end{aligned}
$$

for $i, j=1,2,3$, since $\langle 1,2\rangle+\langle 2,3\rangle+\langle 3,1\rangle=\pi$, i.e.

$$
W_{i, j}=\frac{A(0, i, j)}{A(i, j)} \cdot \frac{\sqrt{-A(i, j) A(0, i, j, 4)}-A\left(\begin{array}{c}
4, i, j \\
0, i, j
\end{array}\right)}{\sqrt{-A(i, j) A(0, i, j, 4)}+A\left(\begin{array}{c}
4, i, j \\
0, i, j
\end{array}\right)}
$$

and

$$
W_{i, 4}=\frac{A\left(\begin{array}{l}
i, 4, j \\
i, 4, k
\end{array}\right)-\sqrt{-A(i, 4) A(i, j, k, 4)}}{A\left(\begin{array}{l}
i, 4, j \\
i, 4, k
\end{array}\right)+\sqrt{-A(i, 4) A(i, j, k, 4)}}
$$

for the complement $\{j, k\}=\{1,2,3,4\}-\{i, 4\}$. Since $d\langle 1,2\rangle=-d\langle 1,3\rangle-d\langle 2,3\rangle$, (3.4) can be expressed as

$$
\begin{aligned}
2 d V(\widehat{\Delta})= & d\langle 1,3\rangle \log W_{1,3} / W_{1,2}+d\langle 2,3\rangle \log W_{2,3} / W_{1,2} \\
& +d\langle 1,4\rangle \log W_{1,4}+d\langle 2,4\rangle \log W_{2,4}+d\langle 3,4\rangle \log W_{3,4} .
\end{aligned}
$$

We want to express the quantities $W_{1,3} / W_{1,2}$ and $W_{2,3} / W_{1,2}$ in terms of the variables $a_{j, k}, 1 \leq j, k \leq 4$. By a conformal change of variables we may assume 
that $v_{4}=(0,0,1) \in \partial D \cap F_{1} \cap F_{2} \cap F_{3}$ and that

$$
\begin{aligned}
& f_{1}=x_{1}, \quad f_{2}=u_{2,1} x_{1}+u_{2,2} x_{2}, \\
& f_{j}=u_{j, 1} x_{1}+u_{j, 2} x_{2}+u_{j, 3} x_{3}+u_{j, 0}, \quad \text { for } j=3,4,
\end{aligned}
$$

where $1=u_{2,1}^{2}+u_{2,2}^{2}=u_{3,1}^{2}+u_{3,2}^{2}=u_{4,1}^{2}+u_{4,2}^{2}+u_{4,3}^{2}-u_{4,0}^{2}$ and $u_{3,3}+u_{3,0}=0$. We can further assume that $u_{2,2}>0, u_{3,2}<0, u_{3,3}<0$ and $u_{4,3}>0$. We then have $a_{0,1}=a_{0,2}=0$ and

LEMMA 3.1

$$
W_{1,2}=\frac{a_{0,3}^{2} A(1,2,4)}{A(1,2,3,4)} .
$$

Pro of. Since

(3.9) $\quad 0=A(0,1,2,3,4)$

$$
=A(1,2,3,4)-a_{0,3}^{2} A(1,2,4)+2 a_{0,3} a_{0,4} A\left(\begin{array}{l}
1,2,3 \\
1,2,4
\end{array}\right)-a_{0,4}^{2} A(1,2,3),
$$

we have

$$
a_{0,4}=\frac{A(1,2,4) a_{0,3}^{2}-A(1,2,3,4)}{2 a_{0,3} A\left(\begin{array}{c}
1,2,3 \\
1,2,4
\end{array}\right)}
$$

from the equality $A(1,2,3)=0$. Since $A(1,2) A(1,2,3,4)-A\left(\begin{array}{l}1,2,3 \\ 1,2,4\end{array}\right)^{2}=0$, we have

$$
\begin{aligned}
& A(0,1,2,4)=A(1,2,4)-A(1,2) a_{0,4}^{2} \\
& =-\frac{A(1,2)\left\{a_{0,3}^{2} A(1,2,4)+A(1,2,3,4)\right\}^{2}}{4 a_{0,3}^{2} A\left(\begin{array}{c}
1,2,3 \\
1,2,4
\end{array}\right)^{2}}, \text { i.e. } \\
& \sqrt{-A(1,2) A(0,1,2,4)}=-\frac{A(1,2)\left\{a_{0,3}^{2} A(1,2,4)+A(1,2,3,4)\right\}}{2 a_{0,3} A\left(\begin{array}{l}
1,2,3 \\
1,2,4
\end{array}\right)} .
\end{aligned}
$$

Note that $a_{0,3}>0, A(1,2)>0, A(1,2,4)<0, A\left(\begin{array}{c}1,2,3 \\ 1,2,4\end{array}\right)>0$ and $A(1,2,3,4)<0$. Again from (3.10),

$$
\sqrt{-A(1,2) A(0,1,2,4)}-A\left(\begin{array}{l}
4,1,2 \\
0,1,2
\end{array}\right)=\frac{-a_{0,3} A(1,2,4) A(1,2)}{A\left(\begin{array}{l}
1,2,3 \\
1,2,4
\end{array}\right)} .
$$

In the same way

$$
\sqrt{-A(1,2) A(0,1,2,4)}+A\left(\begin{array}{l}
4,1,2 \\
0,1,2
\end{array}\right)=-\frac{A(1,2,3,4) A(1,2)}{a_{0,3} A\left(\begin{array}{l}
1,2,3 \\
1,2,4
\end{array}\right)},
$$

whence Lemma 3.1 is proved.

LEMMA 3.2 .

$$
W_{1,3}=a_{0,3}^{2} \frac{A(1,2) A(1,3,4)}{A(1,3) A(1,2,3,4)},
$$




$$
W_{2,3}=a_{0,3}^{2} \frac{A(1,2) A(2,3,4)}{A(2,3) A(1,2,3,4)} .
$$

Proof. First remark $a_{0,3}=u_{3,0}>0, A\left(\begin{array}{l}1,3,2 \\ 1,3,4\end{array}\right)=u_{2,2} u_{3,2} u_{3,3}\left(u_{4,0}+u_{4,3}\right)<0$, $A\left(\begin{array}{l}1,2 \\ 1,3\end{array}\right)=u_{2,2} u_{3,2}<0$ and $A\left(\begin{array}{c}0,1,3,2 \\ 0,1,3,4\end{array}\right)=-u_{2,2} u_{3,3}\left(u_{3,3} u_{4,2}-u_{3,2} u_{4,3}\right)>0$. By the Jacobi identity

$$
\begin{aligned}
0 & =A(0,1,2,3,4) A(0,1,3) \\
& =A(0,1,3,4) A(0,1,2,3)-A\left(\begin{array}{l}
0,1,3,2 \\
0,1,3,4
\end{array}\right)^{2} \\
& =-A(0,1,3,4) A(1,2) a_{0,3}^{2}-A\left(\begin{array}{l}
0,1,3,2 \\
0,1,3,4
\end{array}\right)^{2}
\end{aligned}
$$

since $A(0,1,2,3)=-a_{0,3}^{2} A(1,2)$, whence

$$
A(0,1,3,4)=-\frac{A\left(\begin{array}{l}
0,1,3,2 \\
0,1,3,4
\end{array}\right)^{2}}{a_{0,3}^{2} A(1,2)} .
$$

From (1.3) and the above,

$$
\sqrt{-A(1,3) A(0,1,3,4)}=\sqrt{\frac{A(1,3)}{A(1,2)}} \frac{A\left(\begin{array}{c}
0,1,3,2 \\
0,1,3,4
\end{array}\right)}{a_{0,3}}
$$

where $A\left(\begin{array}{c}0,1,3,2 \\ 0,1,3,4\end{array}\right)$ equals

$$
\frac{1}{2} A\left(\begin{array}{l}
1,3,2 \\
1,3,4
\end{array}\right)-a_{0,3}^{2} \frac{A\left(\begin{array}{l}
1,2 \\
1,4
\end{array}\right) A\left(\begin{array}{l}
1,2,3 \\
1,2,4
\end{array}\right)-\frac{1}{2} A\left(\begin{array}{l}
1,2 \\
1,3
\end{array}\right) A(1,2,4)}{A\left(\begin{array}{l}
1,2,4 \\
1,2,3
\end{array}\right)},
$$

in view of the Jacobi identities $A\left(\begin{array}{l}1,2 \\ 1,3\end{array}\right) A\left(\begin{array}{l}1,2,3 \\ 1,2,4\end{array}\right)=-A(1,2) A\left(\begin{array}{l}1,3,2 \\ 1,3,4\end{array}\right)$ and $A(1,2) A(1,3)-A\left(\begin{array}{l}1,2 \\ 1,3\end{array}\right)^{2}=0$. Hence

$$
\begin{aligned}
\sqrt{-A(1.3) A(0,1,3,4)}+A\left(\begin{array}{l}
4,1,3 \\
0,1,3
\end{array}\right) & \\
= & \sqrt{\frac{A(1,3)}{A(1,2)}} \frac{A\left(\begin{array}{l}
1,3,2 \\
1,3,4
\end{array}\right)}{a_{0,3}}+a_{0,3} \frac{A\left(\begin{array}{l}
1,2,3 \\
1,2,4
\end{array}\right)}{A(1,2)} \\
& =-\frac{A\left(\begin{array}{l}
1,2,3 \\
1,2,4
\end{array}\right)}{A(1,2)}\left\{-\frac{A(1,3)}{a_{0,3}}-a_{0,3}\right\}=\frac{A\left(\begin{array}{l}
1,2,3 \\
1,2,4
\end{array}\right) A(0,1,3)}{a_{0,3} A(1,2)},
\end{aligned}
$$

since $A\left(\begin{array}{l}1,2,3 \\ 1,2,4\end{array}\right)=A\left(\begin{array}{l}1,2 \\ 1,4\end{array}\right) A\left(\begin{array}{l}1,2 \\ 1,3\end{array}\right)-A(1,2) A\left(\begin{array}{l}1,3 \\ 1,4\end{array}\right)$ and $A(0,1,3)=A(1,3)+a_{0,3}^{2}$. Similarly

$$
\sqrt{-A(1,3) A(0,1,3,4)}-A\left(\begin{array}{l}
4,1,3 \\
0,1,3
\end{array}\right)=-a_{0,3} \frac{A(1,2) A(1,3,4)}{A\left(\begin{array}{l}
1,2,4 \\
1,2,3
\end{array}\right)}
$$


Now (3.21) and (3.22) imply

$$
\begin{aligned}
& \frac{\sqrt{-A(1,3) A(0,1,3,4)}-A\left(\begin{array}{l}
4,1,3 \\
0,1,3
\end{array}\right)}{\sqrt{-A(1,3) A(0,1,3,4)}+A\left(\begin{array}{l}
4,1,3 \\
0,1,3
\end{array}\right)} \\
& =-\frac{a_{0,3}^{2} A(1,2)^{2} A(1,3,4)}{A\left(\begin{array}{l}
1,2,4 \\
1,2,3
\end{array}\right)^{2} A(0,1,3)}=\frac{A(1,2) A(1,3,4) a_{0,3}^{2}}{A(1,2,3,4) A(0,1,3)},
\end{aligned}
$$

which proves $(3.15) ;(3.16)$ follows by symmetry.

Corollary.

$$
\begin{aligned}
& W_{1,3} / W_{1,2}=\frac{A(1,2) A(1,3,4)}{A(1,3) A(1,2,4)}, \\
& W_{2,3} / W_{1,2}=\frac{A(1,2) A(2,3,4)}{A(2,3) A(1,2,4)} .
\end{aligned}
$$

As a result we have

Proposition (modified Schläfli formula).

$$
\begin{aligned}
2 d V_{3}(\widehat{\Delta})= & d\langle 1,3\rangle \log \left(W_{1,3} / W_{1,2}\right)+d\langle 2,3\rangle \log \left(W_{2,3} / W_{1,2}\right) \\
& +d\langle 1,4\rangle \log W_{1,4}+d\langle 2,4\rangle \log W_{2,4}+d\langle 3,4\rangle \log W_{3,4},
\end{aligned}
$$

where $W_{1,3} / W_{1,2}$ and $W_{2,3} / W_{1,2}$ are given by $(3.24)-(3.25)$ and $W_{i, 4}$ are given by (3.6)

(2) Suppose that $v_{3}, v_{4} \in \partial D$ and $v_{1}, v_{2} \in D$. Then $A(1,2,3)=A(1,2,4)=0$, or equivalently $\langle 1,2\rangle+\langle 2,3\rangle+\langle 3,1\rangle=\langle 1,2\rangle+\langle 2,4\rangle+\langle 4,1\rangle=\pi$. One can choose as independent variables $\langle 1,3\rangle,\langle 2,3\rangle,\langle 1,4\rangle$ and $\langle 3,4\rangle$, so that

$$
\begin{aligned}
2 d V_{3}(\widehat{\Delta})= & d\langle 1,3\rangle \log \left(W_{2,4} W_{1,3} / W_{1,2}\right)+d\langle 2,3\rangle \log \left(W_{2,4} W_{2,3} / W_{1,2}\right) \\
& +d\langle 1,4\rangle \log \left(W_{1,4} / W_{2,4}\right)+d\langle 3,4\rangle \log \left(W_{3,4}\right) .
\end{aligned}
$$

We must express each coefficient on the right hand side as a function of $a_{j, k}$, $1 \leq j, k \leq 4$. As functions of $\mu$,

$$
\text { C.T. }\left\{\frac{A(1,2)}{A(0,1,2)}\right\}^{-\mu / 2} V_{1}\left(\widehat{\Delta}_{1,2} \mid \mu\right)-2 \log 2=-\log \frac{A(1,2)}{A(0,1,2)}=0,
$$

i.e. $W_{1,2}=1$, since it is assumed that $a_{0,1}=a_{0,2}=0$. As for $W_{1,3}, W_{2,3}$, Lemma 3.2 is valid. For $W_{1,4}$ and $W_{2,4}$, similarly,

$$
\begin{aligned}
W_{1,4} & =a_{0,4}^{2} \frac{A(1,2) A(1,4,3)}{A(1,4) A(1,2,3,4)}, \\
W_{2,4} & =a_{0,4}^{2} \frac{A(1,2) A(2,4,3)}{A(2,4) A(1,2,3,4)} .
\end{aligned}
$$

On the other hand, $W_{3,4}$ equals (3.6). (3.10) reduces to $2 a_{0,3} a_{0,4} A\left(\begin{array}{l}1,2,3 \\ 1,2,4\end{array}\right)=$ 
$-A(1,2,3,4)$. Hence

$$
\begin{aligned}
W_{1,3} W_{2,4} / W_{1,2} & =-\frac{1}{4} \frac{A(1,2) A(1,3,4) A(2,3,4)}{A(2,4) A(1,3) A(1,2,3,4)}, \\
W_{2,4} W_{2,3} / W_{1,2} & =-\frac{1}{4} \frac{A(1,2) A(2,3,4)^{2}}{A(2,4) A(2,3) A(1,2,3,4)}, \\
W_{1,4} / W_{2,4} & =\frac{A(2,4) A(1,3,4)}{A(1,4) A(2,3,4)}, \\
W_{3,4} & =\frac{A\left(\begin{array}{l}
3,4,1 \\
3,4,2
\end{array}\right)-\sqrt{-A(3,4) A(1,2,3,4)}}{\left(\begin{array}{l}
3,4,1 \\
3,4,2
\end{array}\right)+\sqrt{-A(3,4) A(1,2,3,4)}},
\end{aligned}
$$

since $A\left(\begin{array}{l}1,2,3 \\ 1,2,4\end{array}\right)^{2}=-A(1,2) A(1,2,3,4)$.

(3) We assume that $v_{2}, v_{3}, v_{4} \in \partial D$, and $v_{1} \in D$. Then $A(1,2,3)=A(1,2,4)$ $=A(1,3,4)=0$, or equivalently $\langle 1,2\rangle+\langle 2,3\rangle+\langle 3,1\rangle=\langle 1,2\rangle+\langle 2,4\rangle+\langle 4,1\rangle=$ $\langle 1,3\rangle+\langle 3,4\rangle+\langle 4,1\rangle=\pi$. One can choose as independent variables $\langle 1,2\rangle,\langle 1,3\rangle$ and $\langle 1,4\rangle$. (3.7) reduces to

$$
\begin{aligned}
2 d V_{3}(\widehat{\Delta}) & =d\langle 1,2\rangle \log \left(W_{1,2} /\left(W_{2,3} W_{2,4}\right)\right) \\
& +d\langle 1,3\rangle \log \left(W_{1,3} /\left(W_{2,3} W_{3,4}\right)\right)+d\langle 1,4\rangle \log \left(W_{1,4} /\left(W_{2,4} W_{3,4}\right)\right) .
\end{aligned}
$$

By using the relation $2 a_{0,3} a_{0,4}=-A(1,2,3,4) / A\left(\begin{array}{l}1,2,3 \\ 1,2,4\end{array}\right)$, (3.16) and (3.30), we deduce (3.36) below. (3.37) and (3.38) are obtained by symmetry.

$$
\begin{aligned}
& W_{1,2} /\left(W_{2,3} W_{2,4}\right)=-4 \frac{A(2,3) A(2,4) A(1,2,3,4)}{A(1,2) A(2,3,4)^{2}}, \\
& W_{1,3} /\left(W_{2,3} W_{3,4}\right)=-4 \frac{A(2,3) A(3,4) A(1,2,3,4)}{A(1,3) A(2,3,4)^{2}}, \\
& W_{1,4} /\left(W_{3,4} W_{2,4}\right)=-4 \frac{A(3,4) A(2,4) A(1,2,3,4)}{A(1,4) A(2,3,4)^{2}} .
\end{aligned}
$$

(4) Case where all the vertices $v_{1}, v_{2}, v_{3}, v_{4} \in \partial D$. Then $A(i, j, k)$ vanishes for any $i, j, k$, or equivalently $\langle i, j\rangle+\langle j, k\rangle+\langle k, i\rangle=\pi$. One can choose the vertices as $v_{1}=\left(\xi_{1}, \xi_{2}, \xi_{3}\right), v_{2}=(0,1,0), v_{3}=(0,0,-1)$, and $v_{4}=(0,0,1)$ respectively. The point $\left(\xi_{1}, \xi_{2}, \xi_{3}\right)$ in the unit sphere is related to the complex number $z=x+i y$ by stereographic projection:

$$
\xi_{1}=\frac{2 y}{1+|z|^{2}}, \quad \xi_{2}=\frac{2 x}{1+|z|^{2}}, \quad \xi_{3}=\frac{1-|z|^{2}}{1+|z|^{2}} .
$$

Then from (2.2) and (2.5),

$$
d V_{3}(\widehat{\Delta})=\sum_{1 \leq i<j \leq 4} d\langle i, j\rangle W_{i, j},
$$

where $W_{i, j}$ equals $A(0, i, j) / A(i, j)$. Actually $W_{1,2}=\frac{1}{2} W_{1,3}=\frac{1}{2} W_{1,4}=1, W_{2,3}=$ $1+|z|^{2}, W_{2,4}=\left(1+|z|^{2}\right) /|z|^{2}$ and $W_{3,4}=2\left(1+|z|^{2}\right) /|1-z|^{2}$. Moreover, $\langle 1,2\rangle=$ 
$\arg z,\langle 2,3\rangle=\arg \bar{z}(z-1)$ and $\langle 3,1\rangle=\arg (1-\bar{z})$. (3.4) becomes

$$
d V_{3}(\widehat{\Delta})=2(\log |z| d \arg (z-1)-\log |z-1| d \arg z),
$$

i.e. $V_{3}(\widehat{\Delta})$ is the Bloch-Wigner function represented by

$$
V_{3}(\widehat{\Delta})=\frac{1}{i}\left\{\operatorname{dilog} z-\operatorname{dilog} \bar{z}+\log |z| \log \frac{1-z}{1-\bar{z}}\right\} .
$$

This function and its polylogarithmic extension have been investigated by many authors (see [M1], [M2], [G2], [W], [Z]).

Summarizing all the results in Sections 1 and 3, we have

TheOREM. For $v_{1}, \ldots, v_{n+1} \in \partial D \cup D, V_{n}(\widehat{\Delta})$ has a hyperlogarithmic (higher logarithmic) expansion:

$$
V_{n}(\widehat{\Delta})=\sum_{\emptyset \subset I_{1} \subset \ldots \subset I_{\nu-2}} \int_{*}^{A} \theta\left(\begin{array}{c}
\emptyset \\
I_{1}
\end{array}\right) \theta\left(\begin{array}{c}
I_{1} \\
I_{2}
\end{array}\right) \ldots \theta\left(\begin{array}{c}
I_{\nu-3} \\
I_{\nu-2}
\end{array}\right) V_{3}\left(\widehat{\Delta}_{I_{\nu-2}}\right)
$$

for $n=2 \nu-1$, and

$$
V_{n}(\widehat{\Delta})=\sum_{\emptyset \subset I_{1} \subset \ldots \subset I_{\nu-1}} \int_{*}^{A} \theta\left(\begin{array}{c}
\emptyset \\
I_{1}
\end{array}\right) \theta\left(\begin{array}{c}
I_{1} \\
I_{2}
\end{array}\right) \ldots \theta\left(\begin{array}{c}
I_{\nu-2} \\
I_{\nu-1}
\end{array}\right) V_{2}\left(\widehat{\Delta}_{I_{\nu-1}}\right)
$$

for $n=2 \nu$, where $V_{3}\left(\widehat{\Delta}_{J}\right)$ and $V_{2}\left(\widehat{\Delta}_{J}\right)$ are given by (3.26), (3.27), (3.35), (3.41) respectively. $V_{2}\left(\widehat{\Delta}_{J}\right)$ is given by (1.8). $I_{r}=\left\{i_{1}, \ldots, i_{r}\right\}$ denotes a subset of $\{1,2, \ldots, n+1\}$.

4. Appendix. Appell's hypergeometric integrals of type $F_{4}$ and the hyperbolic volume. The integral

$$
\begin{aligned}
& J(\lambda)=\int_{\Delta} \Phi f_{1}^{\lambda_{1}-1} f_{2}^{\lambda_{2}-1} f_{3}^{\lambda_{3}-1} f_{4}^{\lambda_{4}-1} d t_{0} \wedge d t_{1} \wedge d t_{2} \wedge d t_{3} \\
& =\frac{1}{\sqrt{-A(1,2,3,4)}} \int_{y_{1} \geq 0, y_{2} \geq 0, y_{3} \geq 0, y_{4} \geq 0} \exp \left[-\frac{1}{2}^{t} y B y\right] \\
& =\frac{1}{2} \frac{1}{\sqrt{-A(1,2,3,4)}} \Gamma\left(\frac{\lambda_{1}^{\lambda_{1}-1} y_{2}^{\lambda_{2}-1} y_{3}^{\lambda_{3}-1} y_{4}^{\lambda_{4}-1} d \lambda_{3} \wedge d y_{2} \wedge d y_{3} \wedge d y_{4}}{2}\right) \Gamma\left(\lambda_{3}\right) \\
& \quad \times \int_{\eta_{1} \geq 0, \eta_{2} \geq 0} \eta_{1}^{\lambda_{1}-1} \eta_{2}^{\lambda_{3}-1}\left(b_{4,2}+b_{1,2} \eta_{1}+b_{2,3} \eta_{2}\right)^{-\lambda_{2}} \\
& \times\left(b_{4,1} \eta_{1}+b_{4,3} \eta_{2}+b_{1,3} \eta_{1} \eta_{2}\right)^{-\lambda_{4}^{\prime}} d \eta_{1} \wedge d \eta_{2}
\end{aligned}
$$

with $2 \lambda_{4}^{\prime}=\lambda_{1}+\lambda_{2}+\lambda_{4}-\lambda_{3}$, where $B=\left(\left(b_{r, s}\right)\right)_{1 \leq r, s \leq 4}$ denotes the inverse $A^{-1}$. By the definition we have the homogeneity

$$
J\left(\lambda \mid\left\{b_{r, s} \varrho_{r} \varrho_{s}\right\}\right)=\varrho_{1}^{-\lambda_{1}} \varrho_{2}^{-\lambda_{2}} \varrho_{3}^{-\lambda_{3}} \varrho_{4}^{-\lambda_{4}} J\left(\lambda \mid\left\{b_{r, s}\right\}\right),
$$


for $\varrho_{j} \in \mathbb{C}^{*}$. One can choose $\varrho_{r}$ such that $\varrho_{1} \varrho_{2} b_{1,3}=-1, \varrho_{1} \varrho_{4} b_{1,4}=\varrho_{2} \varrho_{4} b_{2,4}=$ $\varrho_{3} \varrho_{4} b_{3,4}=1$. For $b_{1,3}=-1, b_{1,4}=b_{2,4}=b_{3,4}=1, J\left(\lambda \mid\left\{b_{r, s}\right\}\right)$ has an integral expression similar to Appell's hypergeometric function of type $F_{4}$ (see [K1]):

$$
F_{4}\left(\alpha, \beta, \gamma, \gamma^{\prime} \mid u, v\right)=\sum_{l \geq 0, m \geq 0} \frac{(\alpha)_{l+m}(\beta)_{l+m}}{(\gamma)_{l}\left(\gamma^{\prime}\right)_{m}} \frac{u^{l} v^{m}}{l ! m !}
$$

for $u=-b_{1,2}, v=-b_{2,3}, \alpha=\lambda_{2}, \beta=\lambda_{3}, \gamma=1+\left(\lambda_{3}+\lambda_{1}-\lambda_{2}-\lambda_{4}\right) / 2$ and $\gamma^{\prime}=\lambda_{3}-\lambda_{1}+1$ respectively. They both satisfy the following holonomic system of partial differential equations $(\mathcal{E})$ (see [K3], Chap. XI):

$$
\begin{aligned}
u(1-u) R-v^{2} T & -2 u v S \\
+ & \{\gamma-(\alpha+\beta+1) u\} P-(\alpha+\beta+1) v Q-\alpha \beta J=0, \\
v(1-v) T-u^{2} R & -2 u v S \\
+ & \left\{\gamma^{\prime}-(\alpha+\beta+1) v\right\} Q-(\alpha+\beta+1) u P-\alpha \beta J=0
\end{aligned}
$$

for $R=\partial^{2} J / \partial u^{2}, S=\partial^{2} J / \partial u \partial v, T=\partial^{2} J / \partial v^{2}, P=\partial J / \partial u$ and $Q=\partial J / \partial v$. The change of variables

$$
u=w_{1} w_{2}, \quad v=\left(1-w_{1}\right)\left(1-w_{2}\right),
$$

which we call the Burchnall-Chaundy transformation or simply B.C. transformation has an integral representation associated with a line configuration (see [B2], and $[\mathrm{K} 2]$ for an extension):

$$
\begin{array}{r}
F_{4}\left(\alpha, \beta, \gamma, \gamma^{\prime} \mid w_{1} w_{2}^{\prime}, w_{1}^{\prime} w_{2}\right)=\frac{\Gamma(\gamma) \Gamma\left(\gamma^{\prime}\right)}{\Gamma(\alpha) \Gamma(\beta) \Gamma(\gamma-\alpha) \Gamma\left(\gamma^{\prime}-\beta\right)} \\
\times \int_{0}^{1} \int_{0}^{1} x^{\alpha-1} y^{\beta-1}(1-x)^{\gamma-\alpha-1}(1-y)^{\gamma^{\prime}-\beta-1}\left(1-x w_{1}\right)^{\alpha-\gamma-\gamma^{\prime}}\left(1-y w_{2}\right)^{\beta-\gamma-\gamma^{\prime}} \\
\times\left(1-w_{1} x-w_{2} y\right)^{\gamma+\gamma^{\prime}-\alpha-\beta-1} d x \wedge d y,
\end{array}
$$

where we put $w_{1}^{\prime}=1-w_{1}$ and $w_{2}^{\prime}=1-w_{2}$. However, we do not know whether $J(\lambda)$ itself is given by a similar representation through the B.C. transformation.

The holonomic system $(\mathcal{E})$ has an alternative expression, i.e., the Gauss-Manin connection by using the additional integrals $\widetilde{\varphi}(i, j)$ and $\widetilde{\varphi}(1,2,3,4)$. Indeed, we put

$$
\begin{aligned}
\widetilde{\varphi}(i, j) & =\int \Phi \frac{d \tau}{f_{i} f_{j}}, \\
\widetilde{\varphi}(1,2,3,4) & =\int \Phi \frac{d \tau}{f_{1} f_{2} f_{3} f_{4}} .
\end{aligned}
$$

Then as functions of the variables $\left.\left(a_{i, j}\right)\right)_{1 \leq i, j \leq 4}, \widetilde{\varphi}(\emptyset), \widetilde{\varphi}(i, j), \widetilde{\varphi}(1,2,3,4)$ satisfy a variational formula in closed form (Gauss-Manin connection $\left(\mathcal{E}^{\prime}\right)$ ) (see [A3], 
Proposition $\left.2.4_{p}\right)$ :

$$
\begin{aligned}
& d \widetilde{\varphi}(\emptyset)=\frac{1}{2} \sum_{i \neq j} d\langle i, j\rangle \lambda_{i} \lambda_{j} \widetilde{\varphi}(i, j), \\
& A(i, j) d \widetilde{\varphi}(i, j) \\
& =d A\left(\begin{array}{l}
k, i, j \\
l, i, j
\end{array}\right) \lambda_{k} \lambda_{l} \varphi(1,2,3,4)+d a_{i, j} \widetilde{\varphi}(\emptyset) \\
& +\lambda_{k}\left\{-d A\left(\begin{array}{c}
i, j \\
k, j
\end{array}\right) \widetilde{\varphi}(k, j)+d A\left(\begin{array}{c}
i, j \\
k, i
\end{array}\right) \widetilde{\varphi}(k, i)\right\} \\
& +\lambda_{l}\left\{-d A\left(\begin{array}{l}
i, j \\
l, j
\end{array}\right) \widetilde{\varphi}(l, j)+d A\left(\begin{array}{l}
i, j \\
l, i
\end{array}\right) \widetilde{\varphi}(l, i)\right\}, \\
& A(1,2,3,4) d \widetilde{\varphi}(1,2,3,4)=\frac{1}{2} \sum_{i \neq j}(-1)^{i+j} d A\left(\begin{array}{c}
k, i, j \\
l, i, j
\end{array}\right) \widetilde{\varphi}(i, j) \\
& +\frac{1}{2} d A(1,2,3,4)\left\{-1+\lambda_{1}+\lambda_{2}+\lambda_{3}+\lambda_{4}\right\} \widetilde{\varphi}(1,2,3,4),
\end{aligned}
$$

with the fundamental relations

$$
0=\lambda_{j} \widetilde{\varphi}(1,2,3,4)-\sum_{k=1, k \neq j}^{4} b_{k, j} \widetilde{\varphi}(j, k)
$$

for each $j, 1 \leq j \leq 4$. Hence $\widetilde{\varphi}(1,2,3,4), \widetilde{\varphi}(1,4), \widetilde{\varphi}(2,4)$ and $\widetilde{\varphi}(3,4)$ are expressed by linear combinations of $\widetilde{\varphi}(1,2), \widetilde{\varphi}(2,3)$ and $\widetilde{\varphi}(3,1)$ :

(4.14) $2 \lambda_{4} b_{2,3} \widetilde{\varphi}(1,4)$

$$
\begin{aligned}
= & \left(\lambda_{2}+\lambda_{3}+\lambda_{4}-1\right) b_{1,4} \widetilde{\varphi}(2,3)+\left(\lambda_{2}+\lambda_{3}-\lambda_{1}-\lambda_{4}\right) \widetilde{\varphi}(1,3) \\
& +\left(\lambda_{2}+\lambda_{3}-\lambda_{1}-\lambda_{4}\right) b_{3,4} \widetilde{\varphi}(1,2), \quad \text { etc. }
\end{aligned}
$$

The volume $V_{3}(\widehat{\Delta})$ given by the formula

$$
y \int_{\eta_{1} \geq 0, \eta_{2} \geq 0}\left(1+b_{2,3} \eta_{1}+b_{2,1} \eta_{2}\right)^{-1}\left(\eta_{1}+\eta_{2}-\eta_{1} \eta_{2}\right)^{-1} d \eta_{1} \wedge d \eta_{2}
$$

is a special case of the hypergeometric integrals of Appell's type $F_{4}$ for $\alpha=\beta=$ $\gamma=\gamma^{\prime}=1$. The equations $\left(\mathcal{E}^{\prime}\right)$ reduce to $(3.41)$.

The B.C. transformation gives

$$
w, \bar{w}=\frac{1+b_{2,3}-b_{1,2} \pm \sqrt{B(1,2,3,4)}}{2}
$$

for $b_{i, i}=0, b_{1,3}=-1, b_{1,4}=b_{2,4}=b_{3,4}=1$ and

$$
b_{1,2}=-\frac{1-\xi_{2}}{2\left(1+\xi_{3}\right)}, \quad b_{2,3}=-\frac{1-\xi_{3}}{2\left(1+\xi_{3}\right)} .
$$

$B(1,2,3,4)$ equals $1+b_{1,2}^{2}+b_{2,3}^{2}+2 b_{2,3}+2 b_{1,2}-2 b_{1,2} b_{2,3}=-\xi_{1}^{2} /\left(1+\xi_{3}\right)^{2}=y^{2}$. 
On the other hand,

$$
z=\frac{\xi_{2}+i \xi_{1}}{1+\xi_{3}}=1-\frac{1}{w}
$$

Hence the B.C. transformation

$$
w \bar{w}=-b_{1,2}, \quad(1-w)(1-\bar{w})=-b_{2,3}
$$

is the composite of the linear fractional transformation (4.17) and the correspondence (3.39) between the configuration matrix $B$ and the point $z \in \mathbb{C}$ which represents the vertex $v_{1}$.

\section{References}

[A1] K. Aomoto, Fonctions hyper-logarithmiques et groupes de monodromie unipotents, J. Fac. Sci. Univ. Tokyo 25 (1973), 149-156.

[A2] -, On structure of integrals of power product linear functions, Sci. Papers Col. Gen. Edu. Univ. Tokyo 27 (1977), 49-61.

[A3] - Configurations and invariant Gauss-Manin connections of integrals I, Tokyo J. Math. 5 (1982), 249-287; II, ibid. 6 (1983), 1-24.

[A4] —, Vanishing of certain 1-form attached to a configuration, ibid. 9 (1986), 453-455.

[A5] -, Analytic structure of Schläfli function, Nagoya Math. J. 68 (1977), 1-16.

[B1] A. A. Beilinson, A. Varchenko and V. Schechtman, Projective geometry and Ktheory, Algebra and Anal. 2 (1990), 78-130.

[B2] J. L. Burchnall and T. W. Chaundy, Expansions of Appell's double hypergeometric functions, Quart. J. Math. 11 (1940), 249-270.

[G1] I. M. Gelfand and A. V. Zelevinski, Algebraic and combinatorial aspect of general theory of hypergeometric functions, Funct. Anal. Appl. 20 (1986), 17-34.

[G2] A. B. Goncharov, The classical trilogarithm, algebraic K-theory of fields and Dedekind zeta functions, preprint, 1990.

[H1] R. Hain, The geometry of the mixed Hodge structure on the fundamental group, in: Proc. Sympos. Pure Math. 46, Amer. Math. Soc., 1987, 247-282.

[H2] R. Hain and R. MacPherson, Higher logarithms, Illinois J. Math. 34 (1990), 392-475.

[K1] J. Kaneko, Monodromy group of Appell's system (F4), Tokyo J. Math. 4 (1981), 35-54.

[K2] -, Selberg integrals and hypergeometric functions, preprint, 1990.

[K3] M. Kashiwara and P. Schapira, Sheaves on Manifolds, Chap. XI, Springer, 1990.

[K4] R. Kellerhals, On the volume of hyperbolic polyhedra, Math. Ann. 285 (1989), 541-569.

[K5] —, The dilogarithm and volumes of hyperbolic polytopes, preprint.

[M1] J. Milnor, Hyperbolic geometry. The first 150 years, Bull. Amer. Math. Soc. 6 (1982), 9-24.

[M2] -, On polylogarithms, Hurwitz zeta functions, and the Kubert identities, Enseign. Math. 29 (1983), 281-322.

[V] A. Varchenko, Multidimensional hypergeometric functions and their appearance in conformal field theory. Algebraic K-theory, algebraic geometry, etc., congress talk in Kyoto, 1990.

[W] Z. Wojtkowiak, A note on functional equations of polylogarithms, preprint, 1990.

[Z] D. Zagier, Hyperbolic manifolds and special values of Dedekind zeta functions, Invent. Math. 83 (1986), 285-301. 